\title{
APPLICATION AND GRAPHICAL INTERPRETATION OF A NEW TWO-DIMENSIONAL QUATERNION FRACTIONAL FOURIER TRANSFORM
}

\author{
KHINAL PARMAR*, V. R. LAKSHMI GORTY \\ NMIMS, MPSTME, Vile Parle - West, Mumbai - 400056, India \\ *Corresponding author: khinal.parmar@nmims.edu
}

\begin{abstract}
In this paper, a new two-dimensional quaternion fractional Fourier transform is developed. The properties such as linearity, shifting and derivatives of the quaternion-valued function are studied. The convolution theorem and inversion formula are also established. An example with graphical representation is solved. An application related to two-dimensional quaternion Fourier transform is also demonstrated.
\end{abstract}

\section{INTRODUCTION}

In 1853, quaternions were developed by W. R. Hamilton [10]. The necessity of enlarging the operations on three-dimensional vectors to include multiplication and division led Hamilton to introduce the fourdimensional algebra of quaternions. In 1993, Ell [6] introduced quaternion Fourier transform for application to two-dimensional linear time-invariant systems of partial differential equations. In 2001 [3], authors defined non-commutative hypercomplex Fourier transforms of multidimensional Signals. In 2007 [9], author introduced right side quaternion Fourier transform. In 2008 [8], the concept of fractional quaternion Fourier transform was presented. In [11], the author studied the uncertainty principle for the quaternion Fourier transform. Authors in [1] developed quaternion domain Fourier transforms and its application in mathematical statistics. In [4], Plancherel theorem and quaternion Fourier transform for square-integrable functions were studied.

Received April $7^{\text {th }}, 2021$; accepted May 13 ${ }^{\text {th }}, 2021$; published May 25 ${ }^{\text {th }}, 2021$.

2010 Mathematics Subject Classification. 44A35, 44A05, 46S10.

Key words and phrases. quaternion fractional Fourier transform; convolution; operational calculus; graphical representation. (C)2021 Authors retain the copyrights of their papers, and all open access articles are distributed under the terms of the Creative Commons Attribution License. 
Quaternion Fourier transform transfers signals from the real-valued time domain to quaternion-valued frequency domain. But the proposed two-dimensional quaternion fractional Fourier transform will transfer the signal to unified time-frequency domains. Hence, it has a wide range of applications in the field of optics and signal processing.

The organization of the paper is as follows: In section 2, some basic facts of quaternions and quaternionvalued functions are illustrated. In section 3, the two-dimensional quaternion fractional Fourier transform is defined and its inversion formula and operational properties are developed. Graphical interpretation of two-dimensional quaternion fractional Fourier transform is also illustrated. In Section 4, the application of the two-dimensional quaternion fractional Fourier transform is shown.

\section{Preliminary Results}

In quaternions, every element is a linear combination of a real scalar and three imaginary units $\mathbf{i}, \mathbf{j}$ and $\mathbf{k}$ with real coefficients.

Let $q$ be a quaternion defined in

$$
\mathbb{H}=\left\{q=x_{0}+\mathbf{i} x_{1}+\mathbf{j} x_{2}+\mathbf{k} x_{3}: x_{0}, x_{1}, x_{2}, x_{3} \in \mathbb{R}\right\}
$$

be the division ring of quaternions, where $\mathbf{i}, \mathbf{j}, \mathbf{k}$ satisfy Hamilton's multiplication rules (see, e.g. [9])

$$
\mathbf{i j}=-\mathbf{j i}=\mathbf{k}, \mathbf{j k}=-\mathbf{k j}=\mathbf{i}, \mathbf{k i}=-\mathbf{i k}=\mathbf{j}, \mathbf{i}^{2}=\mathbf{j}^{2}=\mathbf{k}^{2}=\mathbf{i j k}=-1
$$

The quaternion conjugate of $q$ is defined by

$$
\bar{q}=x_{0}-\mathbf{i} x_{1}-\mathbf{j} x_{2}-\mathbf{k} x_{3} ; x_{0}, x_{1}, x_{2}, x_{3} \in \mathbb{R}
$$

The norm of $q \in \mathbb{H}$ is defined as

$$
|q|=\sqrt{q \bar{q}}=\sqrt{x_{0}^{2}+x_{1}^{2}+x_{2}^{2}+x_{3}^{2}}
$$

Alternatively, in [13] the quaternions are defined as

$$
\mathbb{H}=\left\{q=q_{1}+j q_{2}: q_{1}, q_{2} \in \mathbb{C}\right\}
$$

where $j$ is the imaginary number satisfying following conditions:

$j^{2}=-1, j r=r j, \forall r \in \mathbb{R}, j i=-i j$, where $i$ is the imaginary number.

From $[13] f \in L^{2}\left(\mathbb{R}^{2} ; \mathbb{H}\right)$, then the function is expressed as

$$
f(u, v)=f_{0}(u, v)+\mathbf{i} f_{1}(u, v)+\mathbf{j} f_{2}(u, v)+\mathbf{k} f_{3}(u, v) .
$$

For some applications the quaternions can be rewritten by replacing $\mathbf{k}$ with $\mathbf{i j}$ as given in [9],

$$
q=x_{0}+\mathbf{i} x_{1}+\mathbf{j} x_{2}+\mathbf{i} x_{3} \mathbf{j}
$$


Another way of rewritting quaternion is

$$
q=x_{+}+x_{-} ; x_{ \pm}=\frac{1}{2}(q \pm \mathbf{i} q \mathbf{j})
$$

$x_{ \pm}$can also be expressed as

$$
x_{ \pm}=\left\{x_{0} \pm x_{3}+\mathbf{i}\left(x_{1} \mp x_{2}\right)\right\} \frac{1 \pm \mathbf{k}}{2}=\frac{1 \pm \mathbf{k}}{2}\left\{x_{0} \pm x_{3}+\mathbf{j}\left(x_{2} \mp x_{1}\right)\right\} .
$$

The real scalar part of the quaternion can be written as [9],

$$
x_{0}=\langle q\rangle_{0}
$$

We can also rewrite the function $f \in L^{2}\left(\mathbb{R}^{2}, \mathbb{H}\right)$ as $[9]$,

$$
f=f_{0}+\mathbf{i} f_{1}+\mathbf{j} f_{2}+\mathbf{i} f_{3} \mathbf{j}
$$

We can also split the function as [9],

$$
f=f_{+}+f_{-} ; f_{+}=\frac{1}{2}(f+\mathbf{i} f \mathbf{j}), f_{-}=\frac{1}{2}(f-\mathbf{i} f \mathbf{j}) .
$$

For $f, g \in L^{2}\left(\mathbb{R}^{2}, \mathbb{H}\right)$ and $\mathbf{u}=(u, v)=u e_{1}+v e_{2} \in \mathbb{R}^{2}$ with $\left\{e_{1}, e_{2}\right\}$ as the basis of $\mathbb{R}^{2}$, the quaternion-valued inner product is defined in [9] as

$$
(f, g)=\int_{\mathbb{R}^{2}} f(\mathbf{u}) \bar{g}(\mathbf{u}) d^{2} \mathbf{u}
$$

with real symmetric part

$$
\langle f, g\rangle=\frac{1}{2}[(f, g)+(g, f)]=\int_{\mathbb{R}^{2}}\langle f(\mathbf{u}) \bar{g}(\mathbf{u})\rangle_{0} d^{2} \mathbf{u} .
$$

The norm of $f \in L^{2}\left(\mathbb{R}^{2}, \mathbb{H}\right)$ is defined as

$$
\|f\|=\sqrt{(f, f)}=\sqrt{\langle f, f\rangle}=\int_{\mathbb{R}^{2}}|f(\mathbf{u})|^{2} d^{2} \mathbf{u}
$$

\section{Main Results}

Definition 3.1. Let $f \in L^{2}\left(\mathbb{R}^{2}, \mathbb{H}\right)$, then two-dimensional quaternion fractional Fourier transform (2DQFrFT) of particular order $\alpha, \beta$ using [9, 12] is defined as

$$
\hat{f}_{\alpha, \beta}\left(w_{1}, w_{2}\right)=F_{\alpha, \beta}\left[f(u, v) ; w_{1}, w_{2}\right]=\int_{-\infty}^{\infty} \int_{-\infty}^{\infty} e^{-\mathbf{i} w_{1}^{\frac{1}{\alpha}} u} f(u, v) e^{-\mathbf{j} w_{2}^{\frac{1}{\beta}} v} d u d v
$$

where $0<\alpha, \beta \leq 1$.

Analogous to [5, page 112], the integral will converge for values of $w_{1}$ and $w_{2}$ in the strips $-s_{1}<\operatorname{Im}\left(w_{1}\right)<s_{1}$ and $-s_{2}<\operatorname{Im}\left(w_{2}\right)<s_{2}$ respectively; where $s_{1}<\operatorname{Re}\left(p_{1}\right), s_{2}<\operatorname{Re}\left(p_{2}\right)$, for $p_{1}=i w_{1}, p_{2}=j w_{2}$. 
The sufficient condition for $f(u, v)$ to have 2D-QFrFT is that $\int_{-\infty}^{\infty} \int_{-\infty}^{\infty}|f(u, v)| d u d v$ exists.

Inversion formula: Consider the inverse formula of quaternion Fourier transform as defined in [9]

$$
f(u, v)=\frac{1}{(2 \pi)^{2}} \int_{-\infty}^{\infty} \int_{-\infty}^{\infty} e^{\mathbf{i} x u} \hat{f}(x, y) e^{\mathbf{j} y v} d x d y
$$

Substituting $x=w_{1}^{\frac{1}{\alpha}}$ and $y=w_{2}^{\frac{1}{\beta}}$.

Then,

$$
\begin{aligned}
& f(u, v)=\frac{1}{(2 \pi)^{2}} \int_{-\infty}^{\infty} \int_{-\infty}^{\infty} e^{\mathbf{i} w_{1}^{\frac{1}{\alpha}} u} w_{1}^{\frac{1}{\alpha}-1} \hat{f}_{\alpha, \beta}\left(w_{1}, w_{2}\right) e^{\mathbf{j} w_{2}^{\frac{1}{\beta}} v} w_{2}^{\frac{1}{\beta}-1} \frac{d w_{1}}{\alpha} \frac{d w_{2}}{\beta} \\
& f(u, v)=\frac{1}{(2 \pi)^{2} \alpha \beta} \int_{-\infty}^{\infty} \int_{-\infty}^{\infty} e^{\mathbf{i} w_{1}^{\frac{1}{\alpha}} u} w_{1}^{\frac{1-\alpha}{\alpha}} \hat{f}_{\alpha, \beta}\left(w_{1}, w_{2}\right) e^{\mathbf{j} w_{2}^{\frac{1}{\beta}} v} w_{2}^{\frac{1-\beta}{\beta}} d w_{1} d w_{2} .
\end{aligned}
$$

Hence, the inversion formula is defined as

$$
\begin{aligned}
& F_{\alpha, \beta}^{-1}\left[\hat{f}_{\alpha, \beta}\left(w_{1}, w_{2}\right)\right]=f(u, v) \\
& =\frac{1}{(2 \pi)^{2} \alpha \beta} \int_{-\infty}^{\infty} \int_{-\infty}^{\infty} e^{\mathbf{i} w_{1}^{\frac{1}{\alpha}} u} w_{1}^{\frac{1-\alpha}{\alpha}} \hat{f}_{\alpha, \beta}\left(w_{1}, w_{2}\right) e^{\mathbf{j} w_{2}^{\frac{1}{\beta}} v} w_{2}^{\frac{1-\beta}{\beta}} d w_{1} d w_{2} .
\end{aligned}
$$

Property 3.1 (Left linearity). For $f_{1}, f_{2} \in L^{2}\left(\mathbb{R}^{2}, \mathbb{H}\right)$ and

$k_{1}, k_{2} \in\left\{q \mid q=x_{0}+\boldsymbol{i} x_{1}, x_{0}, x_{1} \in \mathbb{R}\right\} ;$

$$
F_{\alpha, \beta}\left[k_{1} f_{1}(u, v)+k_{2} f_{2}(u, v)\right]=k_{1} F_{\alpha, \beta}\left[f_{1}(u, v)\right]+k_{2} F_{\alpha, \beta}\left[f_{2}(u, v)\right] .
$$

Proof. For $f_{1}, f_{2} \in L^{2}\left(\mathbb{R}^{2}, \mathbb{H}\right) ; k_{1}, k_{2} \in \mathbb{R}$ and using (3.1), we get

$$
\begin{aligned}
& F_{\alpha, \beta}\left[k_{1} f_{1}(u, v)+k_{2} f_{2}(u, v)\right] \\
& =\int_{-\infty}^{\infty} \int_{-\infty}^{\infty} e^{-\mathbf{i} w_{1}^{\frac{1}{\alpha}} u}\left[k_{1} f_{1}(u, v)+k_{2} f_{2}(u, v)\right] e^{-\mathbf{j} w_{2}^{\frac{1}{\beta}} v} d u d v \\
& =k_{1} \int_{-\infty}^{\infty} \int_{-\infty}^{\infty} e^{-\mathbf{i} w_{1}^{\frac{1}{\alpha}} u}\left[f_{1}(u, v)\right] e^{-\mathbf{j} w_{2}^{\frac{1}{\beta}} v} d u d v \\
& +k_{2} \int_{-\infty}^{\infty} \int_{-\infty}^{\infty} e^{-\mathbf{i} w_{1}^{\frac{1}{\alpha}} u}\left[f_{2}(u, v)\right] e^{-\mathbf{j} w_{2}^{\frac{1}{\beta}} v} d u d v \\
& =k_{1} F_{\alpha, \beta}\left[f_{1}(u, v)\right]+k_{2} F_{\alpha, \beta}\left[f_{2}(u, v)\right] .
\end{aligned}
$$

Property 3.2 (Right linearity). For $f_{1}, f_{2} \in L^{2}\left(\mathbb{R}^{2}, \mathbb{H}\right)$ and $k_{1}^{\prime}, k_{2}^{\prime} \in\left\{q \mid q=x_{0}+\boldsymbol{j} x_{2}, x_{0}, x_{2} \in \mathbb{R}\right\} ;$

$$
F_{\alpha, \beta}\left[f_{1}(u, v) k_{1}^{\prime}+f_{2}(u, v) k_{2}^{\prime}\right]=F_{\alpha, \beta}\left[f_{1}(u, v)\right] k_{1}^{\prime}+F_{\alpha, \beta}\left[f_{2}(u, v)\right] k_{2}^{\prime} .
$$


The proof is similar to property 3.1 .

Property 3.3 (Shifting). For $f \in L^{2}\left(\mathbb{R}^{2}, \mathbb{H}\right)$ and $a, b \in \mathbb{R}$;

$$
F_{\alpha, \beta}[f(u-a, v-b)]=e^{-i w_{1}^{\frac{1}{\alpha}} a} F_{\alpha, \beta}[f(u, v)] e^{-j w_{2}^{\frac{1}{\beta}} b}
$$

Proof. For $f \in L^{2}\left(\mathbb{R}^{2}, \mathbb{H}\right) ; a, b \in \mathbb{R}$ and using (3.1), we get

$$
F_{\alpha, \beta}[f(u-a, v-b)]=\int_{-\infty}^{\infty} \int_{-\infty}^{\infty} e^{-\mathbf{i} w_{1}^{\frac{1}{\alpha}} u} f(u-a, v-b) e^{-\mathbf{j} w_{2}^{\frac{1}{\beta}} v} d u d v
$$

Substituting $u-a=s$ and $v-b=t$ gives

$$
\begin{gathered}
=\int_{-\infty}^{\infty} \int_{-\infty}^{\infty} e^{-\mathbf{i} w_{1}^{\frac{1}{\alpha}}(s+a)} f(s, t) e^{-\mathbf{j} w_{2}^{\frac{1}{\beta}}(t+b)} d s d t \\
=e^{-\mathbf{i} w_{1}^{\frac{1}{\alpha}} a} F_{\alpha, \beta}[f(s, t)] e^{-\mathbf{j} w_{2}^{\frac{1}{\beta}} b} .
\end{gathered}
$$

Property 3.4 (2D-QFrFT of derivatives). For $f \in L^{2}\left(\mathbb{R}^{2}, \mathbb{H}\right)$, the two-dimensional quaternion fractional Fourier transform with derivatives of $f(u, v)$ are as follows:

$$
\text { i) } F_{\alpha, \beta}\left[\frac{\partial}{\partial u} f(u, v)\right]=\left(i w_{1}^{\frac{1}{\alpha}}\right) F_{\alpha, \beta}[f(u, v)] \text {. }
$$

ii) $F_{\alpha, \beta}\left[\frac{\partial}{\partial v} f(u, v)\right]=F_{\alpha, \beta}[f(u, v)]\left(j w_{2}^{\frac{1}{\beta}}\right)$.

In general

$$
\text { iii) } F_{\alpha, \beta}\left[\frac{\partial^{2}}{\partial u \partial v} f(u, v)\right]=\left(\boldsymbol{i} w_{1}^{\frac{1}{\alpha}}\right) F_{\alpha, \beta}[f(u, v)]\left(j w_{2}^{\frac{1}{\beta}}\right) \text {. }
$$

iv) $F_{\alpha, \beta}\left[\frac{\partial^{n}}{\partial u^{n}} f(u, v)\right]=\left(i w_{1}^{\frac{1}{\alpha}}\right)^{n} F_{\alpha, \beta}[f(u, v)]$

$$
\text { v) } F_{\alpha, \beta}\left[\frac{\partial^{n}}{\partial v^{n}} f(u, v)\right]=F_{\alpha, \beta}[f(u, v)]\left(j w_{2}^{\frac{1}{\beta}}\right)^{n}
$$

vi) $F_{\alpha, \beta}\left[\frac{\partial^{n}}{\partial u^{n}} \frac{\partial^{m}}{\partial v^{m}} f(u, v)\right]=\left(\boldsymbol{i} w_{1}^{\frac{1}{\alpha}}\right)^{n} F_{\alpha, \beta}[f(u, v)]\left(j w_{2}^{\frac{1}{\beta}}\right)^{m}$. 
Proof. $i)$ For $f \in L^{2}\left(\mathbb{R}^{2}, \mathbb{H}\right)$, the first order derivative over $f(u, v)$ w.r.t. $u$ is given by

$$
\begin{aligned}
F_{\alpha, \beta}\left[\frac{\partial}{\partial u} f(u, v)\right] \\
=\int_{-\infty}^{\infty} \int_{-\infty}^{\infty} e^{-\mathbf{i} w_{1}^{\frac{1}{\alpha}} u} \frac{\partial}{\partial u} f(u, v) e^{-\mathbf{j} w_{2}^{\frac{1}{\beta}} v} d u d v \\
=\int_{-\infty}^{\infty}\left\{\left[e^{-\mathbf{i} w_{1}^{\frac{1}{\alpha}} u} f(u, v)\right]-\int_{-\infty}^{\infty}-\mathbf{i} w_{1}^{\frac{1}{\alpha}} e^{-\mathbf{i} w_{1}^{\frac{1}{\alpha}} u} f(u, v) d u\right\} e^{-\mathbf{j} w_{2}^{\frac{1}{\beta}} v} d v \\
=\mathbf{i} w_{1}^{\frac{1}{\alpha}} \int_{-\infty}^{\infty} \int_{-\infty}^{\infty} e^{-\mathbf{i} w_{1}^{\frac{1}{\alpha}} u} f(u, v) e^{-\mathbf{j} w_{2}^{\frac{1}{\beta}} v} d u d v \\
=\left(\mathbf{i} w_{1}^{\frac{1}{\alpha}}\right) F_{\alpha, \beta}[f(u, v)] .
\end{aligned}
$$

ii) For $f \in L^{2}\left(\mathbb{R}^{2}, \mathbb{H}\right)$, the first order derivative over $f(u, v)$ w.r.t. $v$ is given by

$$
\begin{aligned}
& F_{\alpha, \beta}\left[\frac{\partial}{\partial v} f(u, v)\right] \\
& =\int_{-\infty}^{\infty} \int_{-\infty}^{\infty} e^{-\mathbf{i} w_{1}^{\frac{1}{\alpha}} u} \frac{\partial}{\partial v} f(u, v) e^{-\mathbf{j} w_{2}^{\frac{1}{\beta}} v} d u d v \\
& =\int_{-\infty}^{\infty} e^{-\mathbf{i} w_{1}^{\frac{1}{\alpha}} u}\left\{\left[f(u, v) e^{-\mathbf{j} w_{2}^{\frac{1}{\beta}} v}\right]-\int_{-\infty}^{\infty} f(u, v) e^{-\mathbf{j} w_{2}^{\frac{1}{\beta}} v}\left(-\mathbf{j} w_{2}^{\frac{1}{\beta}}\right) d v\right\} d u \\
& =\int_{-\infty}^{\infty} \int_{-\infty}^{\infty} e^{-\mathbf{i} w_{1}^{\frac{1}{\alpha}} u} f(u, v) e^{-\mathbf{j} w_{2}^{\frac{1}{\beta}} v} d u d v\left(\mathbf{j} w_{2}^{\frac{1}{\beta}}\right) \\
& =F_{\alpha, \beta}[f(u, v)]\left(\mathbf{j} w_{2}^{\frac{1}{\beta}}\right) .
\end{aligned}
$$

iii) For $f \in L^{2}\left(\mathbb{R}^{2}, \mathbb{H}\right)$, the second order derivative over $f(u, v)$ w.r.t. $u, v$ is given by

$$
\begin{aligned}
& F_{\alpha, \beta}\left[\frac{\partial^{2}}{\partial u \partial v} f(u, v)\right] \\
& =\int_{-\infty}^{\infty} \int_{-\infty}^{\infty} e^{-\mathbf{i} w_{1}^{\frac{1}{\alpha}} u} \frac{\partial^{2}}{\partial u \partial v} f(u, v) e^{-\mathbf{j} w_{2}^{\frac{1}{\beta}} v} d u d v \\
& =\int_{-\infty}^{\infty}\left\{\left[e^{-\mathbf{i} w_{1}^{\frac{1}{\alpha}} u} \frac{\partial}{\partial v} f(u, v)\right]-\int_{-\infty}^{\infty}-\mathbf{i} w_{1}^{\frac{1}{\alpha}} e^{-\mathbf{i} w_{1}^{\frac{1}{\alpha}} u} \frac{\partial}{\partial v} f(u, v) d u\right\} e^{-\mathbf{j} w_{2}^{\frac{1}{\beta}} v} d v \\
& =\left(\mathbf{i} w_{1}^{\frac{1}{\alpha}}\right) \int_{-\infty}^{\infty} \int_{-\infty}^{\infty} e^{-\mathbf{i} w_{1}^{\frac{1}{\alpha}} u} \frac{\partial}{\partial v} f(u, v) e^{-\mathbf{j} w_{2}^{\frac{1}{\beta}} v} d u d v
\end{aligned}
$$




$$
\begin{aligned}
& =\left(\mathbf{i} w_{1}^{\frac{1}{\alpha}}\right) \int_{-\infty}^{\infty} e^{-\mathbf{i} w_{1}^{\frac{1}{\alpha}} u}\left\{\left[f(u, v) e^{-\mathbf{j} w_{2}^{\frac{1}{\beta}} v}\right]-\int_{-\infty}^{\infty} f(u, v) e^{-\mathbf{j} w_{2}^{\frac{1}{\beta}} v}\left(-\mathbf{j} w_{2}^{\frac{1}{\beta}}\right) d v\right\} d u \\
& =\left(\mathbf{i} w_{1}^{\frac{1}{\alpha}}\right) \int_{-\infty}^{\infty} \int_{-\infty}^{\infty} e^{-\mathbf{i} w_{1}^{\frac{1}{\alpha}} u} f(u, v) e^{-\mathbf{j} w_{2}^{\frac{1}{\beta}} v} d u d v\left(\mathbf{j} w_{2}^{\frac{1}{\beta}}\right) \\
& =\left(\mathbf{i} w_{1}^{\frac{1}{\alpha}}\right) F_{\alpha, \beta}[f(u, v)]\left(\mathbf{j} w_{2}^{\frac{1}{\beta}}\right) .
\end{aligned}
$$

iv) By using mathematical induction for $n=1$ by (3.6), we get

$$
F_{\alpha, \beta}\left[\frac{\partial}{\partial u} f(u, v)\right]=\mathbf{i} w_{1}^{\frac{1}{\alpha}} F_{\alpha, \beta}[f(u, v)] .
$$

For $n=2$, the result holds true.

$$
\begin{aligned}
& F_{\alpha, \beta}\left[\frac{\partial^{2}}{\partial u^{2}} f(u, v)\right] \\
& =\int_{-\infty}^{\infty} \int_{-\infty}^{\infty} e^{-\mathbf{i} w_{1}^{\frac{1}{\alpha}} u} \frac{\partial^{2}}{\partial u^{2}} f(u, v) e^{-\mathbf{j} w_{2}^{\frac{1}{\beta}} v} d u d v \\
& =\int_{-\infty}^{\infty}\left\{\left[e^{-\mathbf{i} w_{1}^{\frac{1}{\alpha}} u} \frac{\partial}{\partial u} f(u, v)\right]-\int_{-\infty}^{\infty}-\mathbf{i} w_{1}^{\frac{1}{\alpha}} e^{-\mathbf{i} w_{1}^{\frac{1}{\alpha}} u} \frac{\partial}{\partial u} f(u, v) d u\right\} e^{-\mathbf{j} w_{2}^{\frac{1}{\beta}} v} d v \\
& =\mathbf{i} w_{1}^{\frac{1}{\alpha}} \int_{-\infty}^{\infty} \int_{-\infty}^{\infty} e^{-\mathbf{i} w_{1}^{\frac{1}{\alpha}} u} \frac{\partial}{\partial u} f(u, v) e^{-\mathbf{j} w_{2}^{\frac{1}{\beta}} v} d u d v \\
& =\mathbf{i} w_{1}^{\frac{1}{\alpha}} \int_{-\infty}^{\infty}\left\{\left[e^{-\mathbf{i} w_{1}^{\frac{1}{\alpha}} u} f(u, v)\right]-\int_{-\infty}^{\infty}-\mathbf{i} w_{1}^{\frac{1}{\alpha}} e^{-\mathbf{i} w_{1}^{\frac{1}{\alpha}} u} f(u, v) d u\right\} e^{-\mathbf{j} w_{2}^{\frac{1}{\beta}} v} d v \\
& =\left(\mathbf{i} w_{1}^{\frac{1}{\alpha}}\right)^{2} \int_{-\infty}^{\infty} \int_{-\infty}^{\infty} e^{-\mathbf{i} w_{1}^{\frac{1}{\alpha}} u} f(u, v) e^{-\mathbf{j} w_{2}^{\frac{1}{\beta}} v} d u d v \\
& =\left(\mathbf{i} w_{1}^{\frac{1}{\alpha}}\right)^{2} F_{\alpha, \beta}[f(u, v)] .
\end{aligned}
$$

For $n=k-1$,

$$
\begin{aligned}
& F_{\alpha, \beta}\left[\frac{\partial^{k-1}}{\partial u^{k-1}} f(u, v)\right] \\
& =\int_{-\infty}^{\infty} \int_{-\infty}^{\infty} e^{-\mathbf{i} w_{1}^{\frac{1}{\alpha}} u} \frac{\partial^{k-1}}{\partial u^{k-1}} f(u, v) e^{-\mathbf{j} w_{2}^{\frac{1}{\beta}} v} d u d v \\
& =\int_{-\infty}^{\infty}\left\{\left[e^{-\mathbf{i} w_{1}^{\frac{1}{\alpha}} u} \frac{\partial^{k-2}}{\partial u^{k-2}} f(u, v)\right]-\int_{-\infty}^{\infty}-\mathbf{i} w_{1}^{\frac{1}{\alpha}} e^{-\mathbf{i} w_{1}^{\frac{1}{\alpha}} u} \frac{\partial^{k-2}}{\partial u^{k-2}} f(u, v) d u\right\} e^{-\mathbf{j} w_{2}^{\frac{1}{\beta}} v} d v \\
& =\mathbf{i} w_{1}^{\frac{1}{\alpha}} \int_{-\infty}^{\infty} \int_{-\infty}^{\infty} e^{-\mathbf{i} w_{1}^{\frac{1}{\alpha}} u} \frac{\partial^{k-2}}{\partial u^{k-2}} f(u, v) e^{-\mathbf{j} w_{2}^{\frac{1}{\beta}} v} d u d v .
\end{aligned}
$$


On repeating the integration by parts, we get

$$
F_{\alpha, \beta}\left[\frac{\partial^{k-1}}{\partial u^{k-1}} f(u, v)\right]=\left(\mathbf{i} w_{1}^{\frac{1}{\alpha}}\right)^{k-1} F_{\alpha, \beta}[f(u, v)] .
$$

By method of mathematical induction, the result is true for all $n=k$.

$$
F_{\alpha, \beta}\left[\frac{\partial^{k}}{\partial u^{k}} f(u, v)\right]=\left(\mathbf{i} w_{1}^{\frac{1}{\alpha}}\right)^{k} F_{\alpha, \beta}[f(u, v)] .
$$

Thus, it is true for all $n$.

Similarly, $v$ ) and $v i$ ) can be proved.

Property 3.5 (Power of $u, v)$. For $f \in L^{2}\left(\mathbb{R}^{2}, \mathbb{H}\right)$

$$
\begin{aligned}
& \text { i) } F_{\alpha, \beta}[u f(u, v)]=\left(i \frac{\alpha}{w_{1}^{\frac{1-\alpha}{\alpha}}}\right) \frac{\partial}{\partial w_{1}} F_{\alpha, \beta}[f(u, v)] . \\
& \text { ii) } F_{\alpha, \beta}[v f(u, v)]=\frac{\partial}{\partial w_{2}} F_{\alpha, \beta}[f(u, v)]\left(j \frac{\beta}{w_{2}^{\frac{1-\beta}{\beta}}}\right) .
\end{aligned}
$$

Proof. For $f \in L^{2}\left(\mathbb{R}^{2}, \mathbb{H}\right)$ and using (3.1), we get

$$
\begin{aligned}
& F_{\alpha, \beta}[u f(u, v)]=\int_{-\infty}^{\infty} \int_{-\infty}^{\infty} e^{-\mathbf{i} w_{1}^{\frac{1}{\alpha}} u} u f(u, v) e^{-\mathbf{j} w_{2}^{\frac{1}{\beta}} v} d u d v \\
& =\int_{-\infty}^{\infty} \int_{-\infty}^{\infty}\left(\mathbf{i} \frac{\alpha}{w_{1}^{\frac{1-\alpha}{\alpha}}}\right) \frac{\partial}{\partial w_{1}} e^{-\mathbf{i} w_{1}^{\frac{1}{\alpha}} u} f(u, v) e^{-\mathbf{j} w_{2}^{\frac{1}{\beta}} v} d u d v \\
& =\left(\mathbf{i} \frac{\alpha}{w_{1}^{\frac{1-\alpha}{\alpha}}}\right) \frac{\partial}{\partial w_{1}} \int_{-\infty}^{\infty} \int_{-\infty}^{\infty} e^{-\mathbf{i} w_{1}^{\frac{1}{\alpha}} u} f(u, v) e^{-\mathbf{j} w_{2}^{\frac{1}{\beta}} v} d u d v \\
& =\left(\mathbf{i} \frac{\alpha}{w_{1}^{\frac{1-\alpha}{\alpha}}}\right) \frac{\partial}{\partial w_{1}} F_{\alpha, \beta}[f(u, v)] . \\
& F_{\alpha, \beta}[v f(u, v)]=\int_{-\infty}^{\infty} \int_{-\infty}^{\infty} e^{-\mathbf{i} w_{1}^{\frac{1}{\alpha}} u} v f(u, v) e^{-\mathbf{j} w_{2}^{\frac{1}{\beta}} v} d u d v \\
& =\int_{-\infty}^{\infty} \int_{-\infty}^{\infty} e^{-\mathbf{i} w_{1}^{\frac{1}{\alpha}} u} f(u, v) \frac{\partial}{\partial w_{2}} e^{-\mathbf{j} w_{2}^{\frac{1}{\beta}} v}\left(\mathbf{j} \frac{\beta}{w_{2}^{\frac{1-\beta}{\beta}}}\right) d u d v \\
& =\frac{\partial}{\partial w_{2}} \int_{-\infty}^{\infty} \int_{-\infty}^{\infty} e^{-\mathbf{i} w_{1}^{\frac{1}{\alpha}} u} f(u, v) e^{-\mathbf{j} w_{2}^{\frac{1}{\beta}} v} d u d v\left(\mathbf{j} \frac{\beta}{w_{2}^{\frac{1-\beta}{\beta}}}\right) \\
& =\frac{\partial}{\partial w_{2}} F_{\alpha, \beta}[f(u, v)]\left(\mathbf{j} \frac{\beta}{w_{2}^{\frac{1-\beta}{\beta}}}\right) .
\end{aligned}
$$

Hence the proof. 
Property 3.6 (Power of $\mathbf{i}, \mathbf{j})$. For $f \in L^{2}\left(\mathbb{R}^{2}, \mathbb{H}\right) ; m, n \in \mathbb{N}$

$$
F_{\alpha, \beta}\left[\boldsymbol{i}^{m} f(u, v) \boldsymbol{j}^{n}\right]=\boldsymbol{i}^{m} F_{\alpha, \beta}[f(u, v)] \boldsymbol{j}^{n}
$$

Proof. For $f \in L^{2}\left(\mathbb{R}^{2}, \mathbb{H}\right)$ and using (3.1), we get

$$
\begin{aligned}
& F_{\alpha, \beta}\left[\mathbf{i}^{m} f(u, v) \mathbf{j}^{n}\right]=\int_{-\infty}^{\infty} \int_{-\infty}^{\infty} e^{-\mathbf{i} w_{1}^{\frac{1}{\alpha}} u \mathbf{i}^{m}} f(u, v) \mathbf{j}^{n} e^{-\mathbf{j} w_{2}^{\frac{1}{\beta}} v} d u d v \\
& =\mathbf{i}^{m} \int_{-\infty}^{\infty} \int_{-\infty}^{\infty} e^{-\mathbf{i} w_{1}^{\frac{1}{\alpha}} u} f(u, v) e^{-\mathbf{j} w_{2}^{\frac{1}{\beta}} v} d u d v \mathbf{j}^{n} \\
& =\mathbf{i}^{m} F_{\alpha, \beta}[f(u, v)] \mathbf{j}^{n} .
\end{aligned}
$$

Hence the proof.

Definition 3.2. The convolution for the quaternion valued functions $f, g \in L^{2}\left(\mathbb{R}^{2}, \mathbb{H}\right)$ is defined [14] by

$$
f * g=\int_{-\infty}^{\infty} \int_{-\infty}^{\infty} f(u, v) g(x-u, y-v) d u d v
$$

Theorem 3.7 (Convolution theorem). For $f, g \in L^{2}\left(\mathbb{R}^{2}, \mathbb{H}\right)$;

$$
F_{\alpha, \beta}[f * g]=F_{\alpha, \beta}[f] F_{\alpha, \beta}[g]
$$

Proof. For $f, g \in L^{2}\left(\mathbb{R}^{2}, \mathbb{H}\right) ; X=\left(x_{1}, x_{2}\right), Y=\left(y_{1}, y_{2}\right)$ and $Z=\left(z_{1}, z_{2}\right)$;

$$
\begin{aligned}
F_{\alpha, \beta}[f * g] & =\int_{-\infty}^{\infty} \int_{-\infty}^{\infty} e^{-\mathbf{i} w_{1}^{\frac{1}{\alpha}} x_{1}}(f * g)(X) e^{-\mathbf{j} w_{2}^{\frac{1}{\beta}} x_{2}} d X \\
& =\int_{-\infty}^{\infty} \int_{-\infty}^{\infty} e^{-\mathbf{i} w_{1}^{\frac{1}{\alpha}} x_{1}}\left[\int_{-\infty}^{\infty} \int_{-\infty}^{\infty} f(Y) g(X-Y) d Y\right] e^{-\mathbf{j} w_{2}^{\frac{1}{\beta}} x_{2}} d X \\
& =\int_{-\infty}^{\infty} \int_{-\infty}^{\infty} e^{-\mathbf{i} w_{1}^{\frac{1}{\alpha}} x_{1}} f(Y)\left[\int_{-\infty}^{\infty} \int_{-\infty}^{\infty} g(X-Y) e^{-\mathbf{j} w_{2}^{\frac{1}{\beta}} x_{2}} d X\right] d Y .
\end{aligned}
$$

Substituting $Z=X-Y$, we get

$$
\begin{aligned}
F_{\alpha, \beta}[f * g] & =\int_{-\infty}^{\infty} \int_{-\infty}^{\infty} e^{-\mathbf{i} w_{1}^{\frac{1}{\alpha}}\left(y_{1}+z_{1}\right)} f(Y)\left[\int_{-\infty}^{\infty} \int_{-\infty}^{\infty} g(Z) e^{-\mathbf{j} w_{2}^{\frac{1}{\beta}}\left(y_{2}+z_{2}\right)} d Z\right] d Y \\
& =\left(\int_{-\infty}^{\infty} \int_{-\infty}^{\infty} e^{-\mathbf{i} w_{1}^{\frac{1}{\alpha}} y_{1}} f(Y) e^{-\mathbf{j} w_{2}^{\frac{1}{\beta}} y_{2}} d Y\right)\left(\int_{-\infty}^{\infty} \int_{-\infty}^{\infty} e^{-\mathbf{i} w_{1}^{\frac{1}{\alpha}} z_{1}} g(Z) e^{-\mathbf{j} w_{2}^{\frac{1}{\beta}} z_{2}} d Z\right) \\
& =F_{\alpha, \beta}[f] F_{\alpha, \beta}[g] .
\end{aligned}
$$


Theorem 3.8. The scalar product of two quaternion-valued functions $f, g \in L^{2}\left(\mathbb{R}^{2}, \mathbb{H}\right)$ is given by the scalar product of the corresponding $2 D$-QFrFTs $\hat{f}$ and $\hat{g}$ :

$$
\langle f, g\rangle=\frac{1}{(2 \pi)^{2} \alpha \beta}\left\langle\mathcal{F}_{\alpha, \beta}\left(w_{1}, w_{2}\right), \mathcal{G}_{\alpha, \beta}\left(w_{1}, w_{2}\right)\right\rangle
$$

Proof. For $f, g \in L^{2}\left(\mathbb{R}^{2}, \mathbb{H}\right)$ and using $(2.8)$, we get

$$
\begin{aligned}
& \langle f, g\rangle=\int_{\infty}^{\infty} \int_{\infty}^{\infty}\langle f(u, v) \overline{g(u, v)}\rangle d u d v \\
& =\int_{\infty}^{\infty} \int_{\infty}^{\infty}\left\langle\frac{1}{(2 \pi)^{2} \alpha \beta} \int_{-\infty}^{\infty} \int_{-\infty}^{\infty} e^{\mathbf{i} w_{1}^{\frac{1}{\alpha}} u} w_{1}^{\frac{1-\alpha}{\alpha}} \hat{f}_{\alpha, \beta}\left(w_{1}, w_{2}\right)\right. \\
& \left.\times e^{\mathbf{j} w_{2}^{\frac{1}{\beta}} v} w_{2}^{\frac{1-\beta}{\beta}} d w_{1} d w_{2} \overline{g(u, v)}\right\rangle d u d v \\
& =\frac{1}{(2 \pi)^{2} \alpha \beta} \int_{\infty}^{\infty} \int_{\infty}^{\infty}\left\langle\hat{f}_{\alpha, \beta}\left(w_{1}, w_{2}\right) \int_{-\infty}^{\infty} \int_{-\infty}^{\infty} e^{\mathbf{i} w_{1}^{\frac{1}{\alpha}} u} w_{1}^{\frac{1-\alpha}{\alpha}} e^{\mathbf{j} w_{2}^{\frac{1}{\beta}} v} w_{2}^{\frac{1-\beta}{\beta}}\right. \\
& \times \overline{g(u, v)} d u d v\rangle d w_{1} d w_{2} \\
& =\frac{1}{(2 \pi)^{2} \alpha \beta} \int_{\infty}^{\infty} \int_{\infty}^{\infty}\left\langle\hat{f}_{\alpha, \beta}\left(w_{1}, w_{2}\right) w_{1}^{\frac{1-\alpha}{\alpha}} w_{2}^{\frac{1-\beta}{\beta}}\right. \\
& \left.\times \int_{-\infty}^{\infty} \int_{-\infty}^{\infty} e^{\mathbf{j} w_{2}^{\frac{1}{\beta}} v} \overline{g(u, v)} e^{\mathbf{i} w_{1}^{\frac{1}{\alpha}} u} d u d v\right\rangle d w_{1} d w_{2} \\
& =\frac{1}{(2 \pi)^{2} \alpha \beta} \int_{\infty}^{\infty} \int_{\infty}^{\infty}\left\langle\hat{f}_{\alpha, \beta}\left(w_{1}, w_{2}\right) w_{1}^{\frac{1-\alpha}{\alpha}} w_{2}^{\frac{1-\beta}{\beta}}\right. \\
& \left.\times \overline{-\infty}_{-\infty}^{\infty} \int_{-\infty}^{\infty} e^{-\mathbf{i} w_{1}^{\frac{1}{\alpha}} v} g(u, v) e^{-\mathbf{j} w_{2}^{\frac{1}{\beta}} u} d u d v\right\rangle d w_{1} d w_{2} \\
& =\frac{1}{(2 \pi)^{2} \alpha \beta} \int_{\infty}^{\infty} \int_{\infty}^{\infty}\left\langle w_{1}^{\frac{1-\alpha}{\alpha}} \hat{f}_{\alpha, \beta}\left(w_{1}, w_{2}\right) w_{2}^{\frac{1-\beta}{\beta}} \overline{\hat{g}_{\alpha, \beta}\left(w_{1}, w_{2}\right)}\right\rangle d w_{1} d w_{2} \\
& =\frac{1}{(2 \pi)^{2} \alpha \beta}\left\langle\mathcal{F}_{\alpha, \beta}\left(w_{1}, w_{2}\right), \mathcal{G}_{\alpha, \beta}\left(w_{1}, w_{2}\right)\right\rangle
\end{aligned}
$$

where

$$
\begin{aligned}
& \mathcal{F}_{\alpha, \beta}\left(w_{1}, w_{2}\right)=w_{1}^{\frac{1-\alpha}{\alpha}} \hat{f}_{\alpha, \beta}\left(w_{1}, w_{2}\right) ; \\
& \mathcal{G}_{\alpha, \beta}\left(w_{1}, w_{2}\right)=w_{2}^{\frac{1-\beta}{\beta}} \frac{\hat{g}_{\alpha, \beta}\left(w_{1}, w_{2}\right)}{} .
\end{aligned}
$$

Thus, the theorem holds true. 


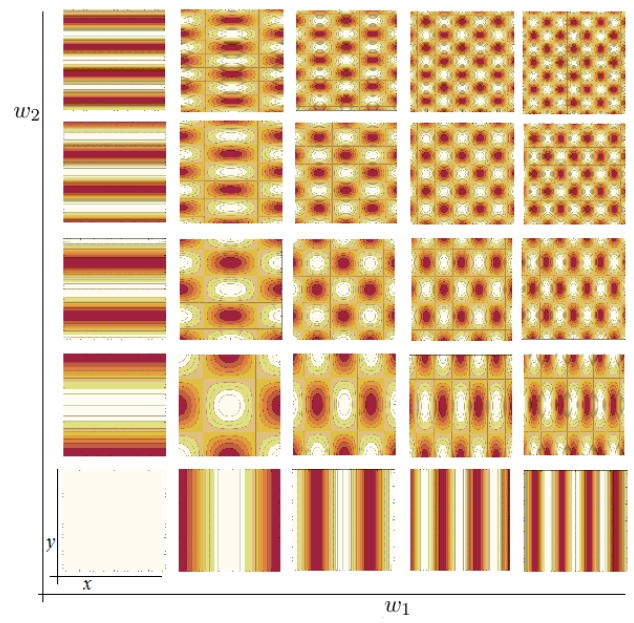

FiguRE 1. Kernel of 2D-QFrFT at $\alpha=1$ and $\beta=1$.

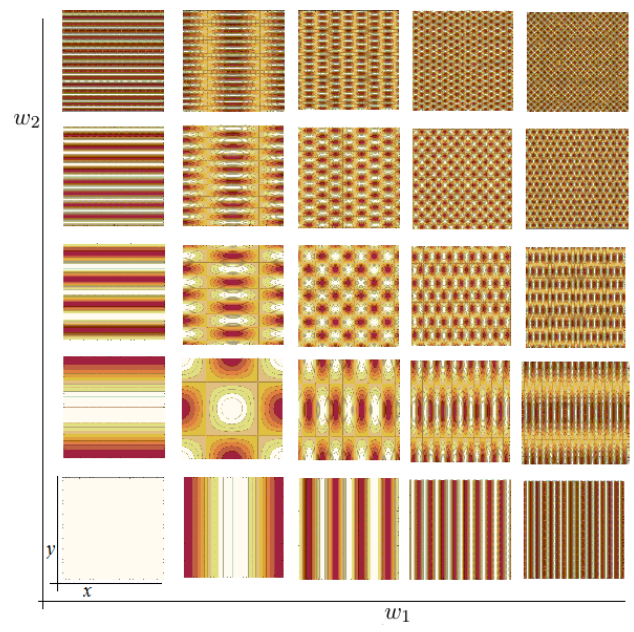

FiguRE 2. Kernel of 2D-QFrFT at $\alpha=1 / 2$ and $\beta=1 / 2$.

Figure 1 shows the kernel of 2D-QFrFT for various values of $w_{1}, w_{2}$ at order $\alpha=1$ and $\beta=1$ which is a particular case of the study developed in this paper. Figure 2 shows the kernel of $2 \mathrm{D}$-QFrFT for various values of $w_{1}, w_{2}$ at order $\alpha=1 / 2$ and $\beta=1 / 2$. For both the figures the range of $x$ and $y$ is between -3 and 3. The 2D-QFrFT is superior in disparity estimation and analyzing genuine $2 \mathrm{D}$ texture as compared to other fractional Fourier transforms and [7]. 

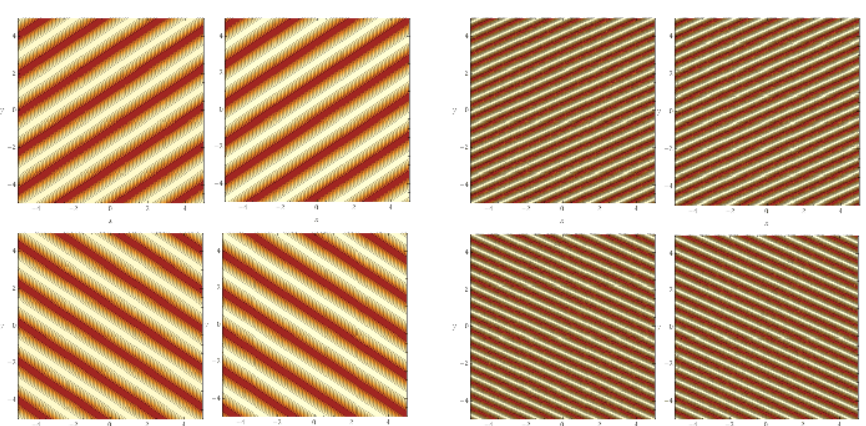

Figure 3. 2D-QFrFT Kernel. Left: Top row: $(1+\mathbf{i j}) / 2$ and $(\mathbf{i}-\mathbf{j}) / 2$ components. Bottom row: $(1-\mathbf{i j}) / 2$ and $(\mathbf{i}+\mathbf{j}) / 2$ components at $\alpha=1, \beta=1 ;$ Right: Top row: $(1+\mathbf{i j}) / 2$ and $(\mathbf{i}-\mathbf{j}) / 2$ components. Bottom row: $(1-\mathbf{i j}) / 2$ and $(\mathbf{i}+\mathbf{j}) / 2$ components at $\alpha=1 / 2, \beta=1 / 2$

The components $(1+\mathbf{i j}) / 2,(\mathbf{i}-\mathbf{j}) / 2,(1-\mathbf{i j}) / 2$ and $(\mathbf{i}+\mathbf{j}) / 2$ are shown in Figure 3 at $\alpha=1, \beta=1$ and $\alpha=1 / 2, \beta=1 / 2$ which represents $2 \mathrm{D}$-QFT extended to $2 \mathrm{D}$-QFrFT. We can also observe the scale-invariant feature of $2 \mathrm{D}-\mathrm{QFrFT}$.

Example 3.1. Find the quaternion fractional Fourier transform of the function:

$$
f(x, y)=\left\{\begin{array}{l}
1 ;|x|<1,|y|<1 \\
0 ; \text { otherwise }
\end{array}\right.
$$

By using (3.1), we get

$$
\begin{gathered}
F_{\alpha, \beta}[f(x, y)]=\int_{-\infty}^{\infty} \int_{-\infty}^{\infty} e^{-\mathbf{i} w_{1}^{\frac{1}{\alpha}} x} f(x, y) e^{-\mathbf{j} w_{2}^{\frac{1}{\beta}} y} d x d y . \\
F_{\alpha, \beta}[f(x, y)]=\int_{-1}^{1} \int_{-1}^{1} e^{-\mathbf{i} w_{1}^{\frac{1}{\alpha}} x} e^{-\mathbf{j} w_{2}^{\frac{1}{\beta}} y} d x d y \\
F_{\alpha, \beta}[f(x, y)]=4 \frac{\sin w_{1}^{\frac{1}{\alpha}}}{w_{1}^{\frac{1}{\alpha}}} \cdot \frac{\sin w_{2}^{\frac{1}{\beta}}}{w_{2}^{\frac{1}{\beta}}} .
\end{gathered}
$$

The graphical representation of the quaternion fractional Fourier transform of the function (3.18) obtained using $\alpha=1$ and $\beta=1$ in (3.19), is now a particular case of (3.1) which is represented in the following figure: 


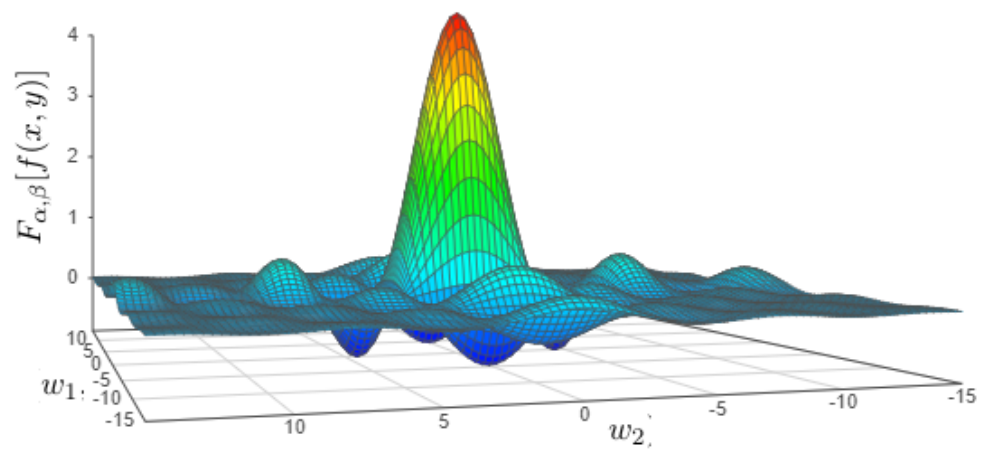

FIGURE 4. Graph of $F_{\alpha, \beta}[f(x, y)]$ with $\alpha=1$ and $\beta=1$.

The graphical representation of the quaternion fractional Fourier transform of the function (3.18) obtained using $\alpha=1 / 2$ and $\beta=1 / 2$ in (3.19) is represented in the following figure:

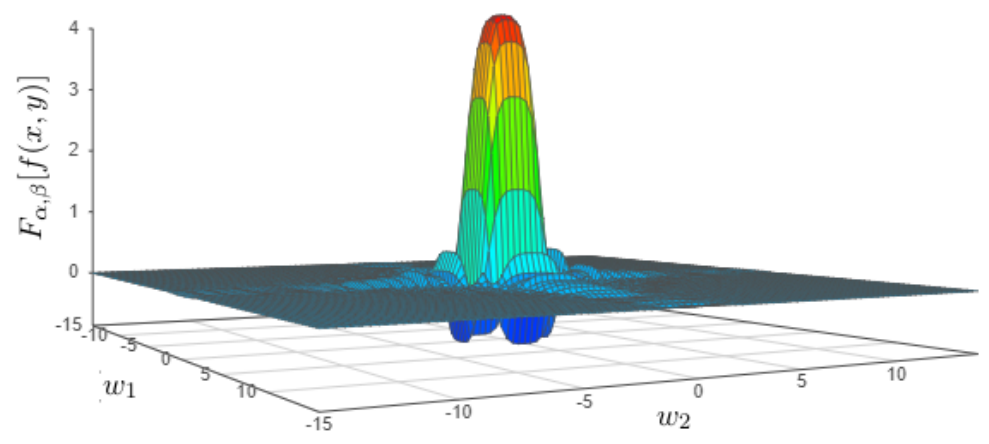

Figure 5. Graph of $F_{\alpha, \beta}[f(x, y)]$ with $\alpha=1 / 2$ and $\beta=1 / 2$.

The graphical representation of the quaternion fractional Fourier transform of the function (3.18) obtained using $\alpha=1 / 2$ and $\beta=1$ in (3.19) is represented in the following figure:

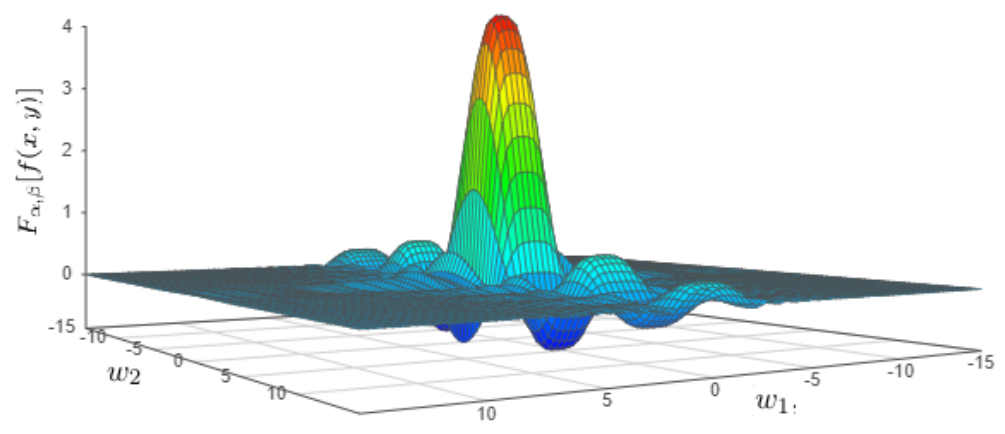

Figure 6 . Graph of $F_{\alpha, \beta}[f(x, y)]$ with $\alpha=1 / 2$ and $\beta=1$. 
Figures 1-3 are plotted using online freeware version of wolframalpha. Figures 4-6 are plotted using online freeware version 3D surface plotter of academo.

\section{Application}

Let us consider the initial value problem from [2]:

$$
\frac{\partial h}{\partial t}-\nabla^{2} h=0, \text { on } \mathbb{R}^{0,2} \times(0, \infty),
$$

and

$$
h(u, v)=f(u, v), f \in \mathcal{S}\left(\mathbb{R}^{0,2} ; \mathbb{H}\right) \text { at } t=0,
$$

where $\mathcal{S}\left(\mathbb{R}^{0,2} ; \mathbb{H}\right)$ is the quaternion Schwartz space and $\nabla^{2}=\frac{\partial^{2}}{\partial^{2} u}+\frac{\partial^{2}}{\partial^{2} v}$.

Applying the definition of 2D-QFrFT to both sides of (4.1), we get

$$
\begin{gathered}
F_{\alpha, \beta}\left[\frac{\partial h}{\partial t}\right]=\left(\mathbf{i} w_{1}^{\frac{1}{\alpha}}\right)^{2} F_{\alpha, \beta}[h]+F_{\alpha, \beta}[h]\left(\mathbf{j} w_{2}^{\frac{1}{\beta}}\right)^{2} \\
\frac{\partial}{\partial t} F_{\alpha, \beta}[h]=-\left(w_{1}^{\frac{2}{\alpha}}+w_{2}^{\frac{2}{\beta}}\right) F_{\alpha, \beta}[h] .
\end{gathered}
$$

The general solution of (4.3) is given by

$$
F_{\alpha, \beta}[h]=C e^{-\left(w_{1}^{\frac{2}{\alpha}}+w_{2}^{\frac{2}{\beta}}\right) t}
$$

where $C$ is a quaternion constant.

By using the initial value condition, we get

$$
F_{\alpha, \beta}[h]=e^{-\left(w_{1}^{\frac{2}{\alpha}}+w_{2}^{\frac{2}{\beta}}\right) t} F_{\alpha, \beta}[f] .
$$

Analogous to [2, equation 6.6], we have

$$
\frac{1}{4 \pi t} F_{\alpha, \beta}\left[e^{-\left(u^{\frac{2}{\alpha}}+v^{\frac{2}{\beta}}\right) / 4 t}\right]=e^{-\left(w_{1}^{\frac{2}{\alpha}}+w_{2}^{\frac{2}{\beta}}\right) t} .
$$

Applying the inversion formula of 2D-QFrFT to (4.5), we get

$$
\begin{aligned}
h & =F_{\alpha, \beta}^{-1}\left[e^{-\left(w_{1}^{\frac{2}{\alpha}}+w_{2}^{\frac{2}{\beta}}\right) t} F_{\alpha, \beta}[f]\right] \\
& =F_{\alpha, \beta}^{-1}\left[\frac{1}{4 \pi t} F_{\alpha, \beta}\left[e^{-\left(u^{\frac{2}{\alpha}}+v^{\frac{2}{\beta}}\right) / 4 t}\right] F_{\alpha, \beta}[f]\right] .
\end{aligned}
$$

Using convolution theorem, we have

$$
h=K_{t} * f
$$


where $K_{t}=\frac{1}{4 \pi t} e^{-\left(u^{\frac{2}{\alpha}}+v^{\frac{2}{\beta}}\right) / 4 t}$.

\section{Conclusion}

The authors developed a new two-dimensional quaternion fractional Fourier transform in this study. The properties such as linearity, shifting and derivatives of the quaternion-valued function are demonstrated. The convolution theorem and inversion formula are also established. An example is illustrated with graphical representation. In the concluding section, an application related to the two-dimensional quaternion Fourier transform is also demonstrated.

Conflicts of Interest: The author(s) declare that there are no conflicts of interest regarding the publication of this paper.

\section{REFERENCES}

[1] M. Bahri, A.K. Amir, C. Lande, The quaternion domain Fourier transform and its application in mathematical statistics, IAENG Int. J. Appl. Math. 48 (2018), 1-7.

[2] M. Bahri, R. Ashino, R. Vaillancourt, Continuous quaternion Fourier and wavelet transforms, Int. J. Wavelets Multiresolut. Inf. Process. 12 (2014), 1460003.

[3] T. Bülow, M. Felsberg, G. Sommer, Non-commutative hyper complex Fourier transforms of multidimensional signals, In: G. Sommer (eds) Geometric Computing with Clifford Algebras, Springer, Berlin, Heidelberg, 2001.

[4] D. Cheng, K.I. Kou, Plancherel theorem and quaternion Fourier transform for square integrable functions, Complex Var. Elliptic Equ. 64 (2019), 223-242.

[5] B. Davies, Integral transforms and their Applications, Springer, New York, 1978.

[6] T.A. Ell, Quaternion-Fourier transforms for analysis of two-dimensional linear time-invariant partial differential systems, in: Proceedings of 32nd IEEE Conference on Decision and Control, IEEE, San Antonio, TX, USA, 1993: pp. $1830-1841$.

[7] T.A. Ell, S.J. Sangwine, Hypercomplex Fourier Transforms of Color Images, IEEE Trans. Image Process. 16 (2007), $22-35$.

[8] X. Guanlei, W. Xiaotong, X. Xiaogang, Fractional quaternion Fourier transform, convolution and correlation, Signal Processing. 88 (2008), 2511-2517.

[9] E.M.S. Hitzer, Quaternion Fourier Transform on Quaternion Fields and Generalizations, Adv. Appl. Clifford Alg. 17 (2007), 497-517.

[10] A.C. Lewis, William Rown Hamilton, Lectures on quaternions (1853), in: Landmark Writings in Western Mathematics 1640-1940, Elsevier, 2005: pp. 460-469.

[11] P. Lian, Uncertainty principle for the quaternion Fourier transform, J. Math. Anal. Appl. 467 (2018), $1258-1269$.

[12] L. Romero, R. Cerutti, L. Luque, A new Fractional Fourier Transform and convolutions products, Int. J. Pure Appl. Math. 66 (2011), 397-408.

[13] R. Roopkumar, Quaternionic one-dimensional fractional Fourier transform, Optik, 127 (2016), 11657-11661.

[14] A.H. Zemanian, Generalized integral transformations, John Wiley Sons Inc., New York, 1968. 